\title{
The Cauchy Problem for the Incompressible 2D-MHD with Power Law-Type Nonlinear Viscous Fluid
}

\author{
Jae-Myoung Kim \\ Department of Mathematics Education, Andong National University, Andong 36729, Republic of Korea
}

Correspondence should be addressed to Jae-Myoung Kim; jmkim02@anu.ac.kr

Received 21 December 2020; Revised 23 February 2021; Accepted 8 March 2021; Published 16 March 2021

Academic Editor: Maria L. Gandarias

Copyright (C) 2021 Jae-Myoung Kim. This is an open access article distributed under the Creative Commons Attribution License, which permits unrestricted use, distribution, and reproduction in any medium, provided the original work is properly cited.

We investigate a motion of the incompressible 2D-MHD with power law-type nonlinear viscous fluid. In this paper, we establish the global existence and uniqueness of a weak solution $(u, b)$ depending on a number $q$ in $\mathbb{R}^{2}$. Moreover, the energy norm of the weak solutions to the fluid flows has decay rate $(1+t)^{-1 / 2}$.

\section{Introduction}

In this paper, we study the weak solutions to the incompressible 2D-MHD with power law-type nonlinear viscous fluid:

$$
\left\{\begin{array}{l}
u_{t}-\nabla \cdot S+(u \cdot \nabla) u+\nabla \pi=(b \cdot \nabla) b, \\
b_{t}-\triangle b+(u \cdot \nabla) b-(b \cdot \nabla) u=0, \\
\operatorname{div} u=0, \operatorname{div} b=0,
\end{array}\right.
$$

Here, $u: \mathbb{R}^{2} \times(0, T) \rightarrow \mathbb{R}^{2}$ is the flow velocity vector, $b$ $: \mathbb{R}^{2} \times(0, T) \rightarrow \mathbb{R}^{2}$ is the magnetic vector, and $\pi: \mathbb{R}^{2} \times(0$, $T) \rightarrow \mathbb{R}$ is the total pressure. We consider the initial value problem of (1), which requires initial conditions:

$$
\begin{gathered}
u(x, 0)=u_{0}(x), \\
b(x, 0)=b_{0}(x) x \in \mathbb{R}^{2} .
\end{gathered}
$$

We assume that the initial data $u_{0}(x), b_{0}(x) \in L^{2}\left(\mathbb{R}^{2}\right)$ hold the incompressibility, i.e., $\nabla \cdot u_{0}(x)=0$ and $\nabla \cdot b_{0}(x)=0$, respectively. In this paper, we deal with $S$ given as

$$
S:=S(D u)=\left(\mu_{0}+\mu_{1}|D u|^{2}\right)^{(q-2) / 2} D u, \quad D u=\frac{\nabla u+\nabla u^{\perp}}{2}
$$

where $\mu_{0} \geq 0$ and $\mu_{1}>0$ are constants (see, e.g., $[1,2]$ ).

In modern industrial application, non-Newtonian fluids play an important role (see [3-5]). In particular, equation (1) is the simplest self-consistent model which describes the dynamics of electrically conducting liquid with involved rheological structure in a magnetic field.

Some examples of non-Newtonian fluids are coal-water, glues, soaps, etc. (see, e.g., [6]). One class of nonNewtonian fluids can be defined by $S=\mu(|D|) D$ ( $D$ is the rate of the strain tensor, $\mu(\cdot)>0$ a real function). That is, the relation between the shear stress and the strain rate is nonlinear. In this paper, we study the case $\mu(s)=\mu_{0}+\mu_{1}$ $s^{q-2}$ which is called power law fluids. Commonly, the case of $q>2$ describes dilatant (or shear thickening) fluids whose viscosity increases with the rate of shear (see, e.g., [6]). On the other hand, pseudoplastic (or shear thinning) fluids correspond to the case of $1<q<2$, where viscosity decreases with the increasing rate of shear (see, e.g., [1]).

In what follows, we review some known results related to our concerns. For incompressible Navier-Stokes equation for a non-Newtonian type, namely, $b=0$ in (1), the existence of weak solutions for $(3 n+2) /(n+2) \leq q$ was first obtained in $[7,8]$, which is unique for $(n+2) / 2 \leq q$ for any dimension 
$n$ (cf. [9]). Later, the existence of weak solutions was investigated for $2 n /(n+2)<q$ in $[10,11]$. On the other hand, in the case of $q=2$, that is, $S(D u)=D u$ and $n=3$, numerous results are known. Among them, we only mention that Ferreira and Villamizar-Roa [12] showed well-posedness, time decay, and stability for 3D magnetohydrodynamic equations.

In $[13,14]$, Samokhin first studied a nonstationary system of equations describing the motion of the Ostwald-de Waele media type and showed a unique existence of a generalized solution for $q \geq 1+(2 n /(n+2))$ to the problem based on the Faedo-Galerkin method and the monotone operator method. Later on, Gunzburger et al. in [15] proved the global unique solvability of the initial boundary value problem for the modified Navier-Stokes equations coupled with the Maxwell equations. Here, the authors use the strain tension containing the diffusion operator; that is, they do not deal with the degenerate power law fluids. Recently, Razafimandimby [16] proved the existence of weak solutions for $q \in(1,(2 n+$ $6) /(n+2)]$ to this model of bipolar type.

In this paper, we will prove the global-in-time existence and uniqueness of the weak solutions for the incompressible 2D-MHD with power law-type nonlinear viscous fluid (1)-(2) under a condition on the range of $q$.

Our results are based on the standard Galerkin method and some uniform estimates.

Denote $\mathbb{M}_{\text {sym }}^{2}$ by the vector space of all symmetric $2 \times 2$ matrices $\zeta=\left(\zeta_{i j}\right)_{1 \leq i, j \leq 2}$. Let $S:=|D u|^{q-2} D u$ and $1 \leq q<\infty$. The deviatoric stress tensor $S=\left(S_{i j}\right), i, j=1,2$, satisfies the following conditions:

(i) $S: Q_{T} \times \mathbb{M}_{\text {sym }}^{2} \rightarrow \mathbb{M}_{\text {sym }}^{3}$ is a Carathéodory function

(ii) Symmetry: $S_{i j}=S_{j i}$

(iii) Polynomial growth:

$$
\left|S_{i j}(\xi)\right| \leq\left(\mu_{0}+\mu_{1}|\xi|^{q-2}\right)|\xi|
$$

(iv) Coercivity condition: there exists $c_{1}>1$ such that

$$
\left(\mu_{0}+\mu_{1}|\xi|^{q-2}\right)|\eta|^{2} \leq \frac{\partial S_{i j}}{\partial \xi_{k l}} \eta_{k l} \eta_{i j} \leq c_{1}\left(\mu_{0}+\mu_{1}|\xi|^{q-2}\right)|\eta|^{2}
$$

(v) Strict monotonicity: for all $\zeta, \eta \in \mathbb{M}_{\text {sym }}^{2}(\zeta \neq \eta)$, $S(\zeta)-S(\eta):(\zeta-\eta)>0$

By the weak solution of the incompressible 2D-MHD with power law-type nonlinear viscous fluid, we mean solutions satisfying the following definitions:
Definition 1 (weak solution). Let $\mu_{0} \geq 0, \mu_{1}>0$, and $q \in(1, \infty)$ . Suppose that $u_{0}, b_{0} \in L^{2}\left(\mathbb{R}^{2}\right)$. We say that $(u, b)$ is a weak solution of the incompressible 2D-MHD with power lawtype nonlinear viscous fluid (1)-(2) if $u$ and $b$ satisfy the following:

$$
\begin{gathered}
u \in L^{\infty}\left([0, T) ; L^{2}\left(\mathbb{R}^{2}\right)\right) \cap L^{2}\left([0, T) ; W^{1, q}\left(\mathbb{R}^{2}\right)\right) \\
b \in L^{\infty}\left([0, T) ; L^{2}\left(\mathbb{R}^{2}\right)\right) \cap L^{2}\left([0, T) ; H^{1}\left(\mathbb{R}^{2}\right)\right)
\end{gathered}
$$

(i) $(u, b)$ satisfies (1) in the sense of distribution; that is,

$$
\begin{gathered}
\int_{0}^{T} \int_{\mathbb{R}^{2}}\left(\frac{\partial \phi}{\partial t}+(u \cdot \nabla) \phi\right) u d x d t+\int_{0}^{T} \int_{\mathbb{R}^{2}} S(D u): \nabla \phi d x d t \\
=\int_{\mathbb{R}^{2}} u_{0} \phi(x, 0) d x+\int_{0}^{T} \int_{\mathbb{R}^{2}}(b \cdot \nabla) \phi b d x d t, \\
\int_{0}^{T} \int_{\mathbb{R}^{2}}\left(\frac{\partial \phi}{\partial t}+\Delta \phi+(u \cdot \nabla) \phi\right) b d x d t \\
=\int_{\mathbb{R}^{2}} b_{0} \phi(x, 0) d x+\int_{0}^{T} \int_{\mathbb{R}^{2}}(b \cdot \nabla) \phi u d x d t,
\end{gathered}
$$

for all $\phi \in C_{0}^{\infty}\left(\mathbb{R}^{2} \times[0, T)\right)$ with $\operatorname{div} \phi=0$, and

$$
\int_{\mathbb{R}^{2}} u \cdot \nabla \psi d x=0, \int_{\mathbb{R}^{2}} b \cdot \nabla \psi d x=0
$$

for every $\psi \in C_{0}^{\infty}\left(\mathbb{R}^{2}\right)$.

Theorem 2. Let $2<q<\infty$ and $\mu_{0} \geq 0$ and $\mu_{1}>0$. Assume that $u_{0}, b_{0} \in L^{2}\left(\mathbb{R}^{2}\right)$. A weak solution $(u, b)$ of the incompressible $2 D-M H D$ with power law-type nonlinear viscous fluid (1)-(2) exists. In particular, in the case $\mu_{0}>0$ and $\mu_{1}>0$, the weak solution $(u, b)$ is unique. Moreover, we obtain the following decay rate of the weak solution:

$$
\|u(t)\|_{L^{2}}+\|b(t)\|_{L^{2}} \leq C(1+t)^{-1 / 2} .
$$

\section{Preliminaries}

In this section, we introduce the notation. Let $I$ be a finite time interval. For $1 \leq q \leq \infty$, we denote by $W^{k, q}\left(\mathbb{R}^{2}\right)$ the usual Sobolev spaces, namely, $W^{k, q}\left(\mathbb{R}^{2}\right)=\left\{f \in L^{q}\left(\mathbb{R}^{2}\right): D^{\alpha} f\right.$ $\left.\in L^{q}\left(\mathbb{R}^{2}\right), 0 \leq|\alpha| \leq k\right\}$. The set of the $q$-th power Lebesgue integrable functions on $\mathbb{R}^{2}$ is denoted by $L^{q}\left(\mathbb{R}^{2}\right)$, and $L_{\text {loc }}^{q}($ $\mathbb{R}^{2}$ ) indicates the set of the locally $q$-th power Lebesgue integrable functions defined on $\mathbb{R}^{2}$. For a function $f(x, t), \mathcal{O} \subset \mathbb{R}^{2}$ , and $J \subset I$, we denote $\|f\|_{L_{x, t}^{p, q}(\mathcal{O} \times J)}=\|\| f\left\|_{L^{p}(\mathcal{O})}\right\|{L^{q}(J)}$. For vector fields $u$, $v$, we write $\left(u_{i} v_{j}\right)_{i, j=1,2,3}$ as $u \otimes v$. We denote $A$ $: B=a_{i j} b_{i j}$ for $3 \times 3$ matrices $A=\left(a_{i j}\right), B=\left(b_{i j}\right)$. The letter $C$ is used to represent a generic constant, which may change from line to line. 
Before looking for a solution for the system (1), we give a lemma.

Lemma 3. Let $(u, b)$ be a solution to the initial value problem of (1)-(2) with the initial data $u_{0}, b_{0} \in L^{2}\left(\mathbb{R}^{2}\right) \cap L^{1}\left(\mathbb{R}^{2}\right)$. Then, we have for $2 \leq p$

$$
\begin{aligned}
|\widehat{u}(\xi, t)|+|\widehat{b}(\xi, t)| \leq & C\left(\widehat{u}_{0}(\xi)+\left|\widehat{b}_{0}(\xi)\right|+|\xi| \int_{0}^{t}\right. \\
& \cdot\left(\|u(s)\|_{L^{2}\left(\mathbb{R}^{2}\right)}^{2}+\|b(s)\|_{L^{2}\left(\mathbb{R}^{2}\right)}^{2}\right) d s \\
& +C|\xi|\left(\int_{0}^{t}\|u(s)\|_{L^{2}\left(\mathbb{R}^{2}\right)} d s\right)^{1 /(p-1)}
\end{aligned}
$$

where $C$ depends only on the $\left(L^{2} \cap L^{1}\right)\left(\mathbb{R}^{2}\right)$-norm of $u_{0}$ and $b_{0}$

Proof. The proof is easily checked. Indeed, it is almost the same as that in [17] replacing (2.5) in [17] by

$$
\begin{aligned}
\int_{0}^{t}\|\nabla u(s)\|_{L^{p-1}\left(\mathbb{R}^{2}\right)}^{p-1} d s \leq & \int_{0}^{t}\|u(s)\|_{L^{2}\left(\mathbb{R}^{2}\right)}^{1 /(p-1)}\|\nabla u(s)\|_{L^{p}\left(\mathbb{R}^{2}\right)}^{p(p-2) /(p-1)} d s \\
\leq & C\left(\int_{0}^{t}\|u(s)\|_{L^{2}\left(\mathbb{R}^{2}\right)} d s\right)^{1 /(p-1)} \\
& \cdot\left(\int_{0}^{t}\|\nabla u(s)\|_{L^{p}\left(\mathbb{R}^{2}\right)}^{p} d t\right)^{(p-2) /(p-1)} \\
\leq & C\left(\int_{0}^{t}\|u(s)\|_{L^{2}\left(\mathbb{R}^{2}\right)} d s\right)^{1 /(p-1)}, \quad p>2 .
\end{aligned}
$$

\section{Proof of Theorem 2}

In this paper, we assume that $\mu_{0}=0$ and $\mu_{1}=1$ for convenience. Let

$$
V_{q}:=\left\{\varphi \in D^{\prime}\left(\mathbb{R}^{2}\right)^{2}: \nabla \cdot \varphi=0\right\}
$$

with $\|\varphi\|_{V_{q}}:=\|D \varphi\|_{L^{q}\left(\mathbb{R}^{2}\right)}$. Now, we will construct the existence of a weak solution to the system (1) via the standard Galerkin method. For this, first of all, we need to find a countable dense subset of the space $\left\{\varphi \in \mathscr{D}\left(\mathbb{R}^{2}\right): \nabla \cdot \varphi=0\right\}$ in $W^{2,2}\left(\mathbb{R}^{2}\right) \cap V_{q}$ in Lemma 3.10 of [18].

Now, we consider Galerkin approximate solutions $u^{m}(t$ )$=\sum_{i=1}^{m} g_{j}^{m}(t) \varphi_{j}$ and $b^{m}(t)=\sum_{i=1}^{m} h_{j}^{m}(t) \psi_{j}$, where the $\varphi_{j}, \psi_{j}$ are the eigenfunctions which are chosen by using Lemma 3.10 of [18].

$$
\begin{gathered}
\left(u_{t}^{m}-\nabla \cdot S(D u)+\left(u^{m} \cdot \nabla\right) u^{m}-\left(b^{m} \cdot \nabla\right) b^{m}, \varphi\right)=0, \\
\left(b_{t}^{m}-\triangle b^{m}+\left(u^{m} \cdot \nabla\right) b^{m}-\left(b^{m} \cdot \nabla\right) u^{m}, \psi\right)=0
\end{gathered}
$$

for $\varphi \in \operatorname{span}\left\{\varphi_{1}, \varphi_{2}, \cdots \varphi_{m}\right\}$ and $\psi \in \operatorname{span}\left\{\psi_{1}, \psi_{2}, \cdots \psi_{m}\right\}$. The initial conditions were

$$
u^{m}(x, 0)=\sum_{i=1}^{m} a_{i} \varphi^{i}(x), b^{m}(x, 0)=\sum_{i=1}^{m} c_{i} \psi^{i}(x)
$$

where $a_{i}=\int_{\mathbb{R}^{2}} u^{m}(x, 0) \cdot \varphi^{i}(x)$ and $c_{i}=\int_{\mathbb{R}^{2}} b^{m}(x, 0) \cdot \psi^{i}(x)$. Indeed, the functions $g_{j}^{m}(t)$ and $h_{j}^{m}(t)$ satisfy the following ordinary differential equations as follows:

$$
\begin{aligned}
& \dot{g}_{j}^{m}(t)+\lambda_{u} g_{j}^{m}(t)+\left(g_{k}^{m}(t)\right)^{p-1} \int_{\mathbb{R}^{2}}\left|\nabla \varphi^{k}\right|^{p-2} \nabla \varphi^{k} \cdot \nabla \varphi^{j} \\
& \quad+g_{k}^{m}(t) g_{l}^{m}(t) \int_{\mathbb{R}^{2}}\left(\varphi^{k} \cdot \nabla \varphi^{l}\right) \varphi^{j}-h_{k}^{m}(t) h_{l}^{m}(t) \int_{\mathbb{R}^{2}}\left(\psi^{k} \cdot \nabla \psi^{l}\right) \varphi^{j}=0
\end{aligned}
$$

$$
\begin{gathered}
\dot{h}_{j}^{m}(t)+\lambda_{b} h_{j}^{m}(t)+g_{k}^{m}(t) h_{l}^{m}(t) \int_{\mathbb{R}^{2}}\left(\varphi^{k} \cdot \nabla \psi^{l}\right) \psi^{j} \\
-h_{k}^{m}(t) g_{l}^{m}(t) \int_{\mathbb{R}^{2}}\left(\psi^{k} \cdot \nabla \varphi^{l}\right) \psi^{j}=0 .
\end{gathered}
$$

By the Carathéodory theorem (see [19], Theorem 3.4 in Appendix), there exist $T_{m}$ such that equation (16) has unique solutions on $\left[0, T_{m}\right)$. Now set $T_{m}=T, T<\infty$.

Proof of Theorem 2. For a proof of existence for a weak solution, we assume that $\mu_{0}=0$ because it is easier for $\mu_{0}>0$.

Part A: existence

Multiplying equation (13) by $u^{m}$ and equation (14) by $b^{m}$ and summing up the equations, we have

$$
\begin{aligned}
& \left\|u^{m}(T)\right\|_{L^{2}\left(\mathbb{R}^{2}\right)}^{2}+\left\|b^{m}(T)\right\|_{L^{2}\left(\mathbb{R}^{2}\right)}^{2}+\left\|\nabla u^{m}\right\|_{L^{q}\left(Q_{T}\right)}^{q}+\left\|\nabla b^{m}\right\|_{L^{2}\left(Q_{T}\right)}^{2} \\
& \quad \leq\left\|u^{m}(0)\right\|_{L^{2}\left(\mathbb{R}^{2}\right)}^{2}+\left\|b^{m}(0)\right\|_{L^{2}\left(\mathbb{R}^{2}\right)}^{2},
\end{aligned}
$$

where we use the divergence free condition, Korn's inequality, and vector identity for the magnetic vector field $b$. For the distributive time derivative $d u^{m} / d t$, we have $\left(d u^{m} / d t\right) \epsilon$ $L^{q^{\prime}}\left(0, T ;\left(W^{1, q}\right)^{*}\right)+L^{q}\left(0, T ;\left(W^{1, q^{\prime}}\right)^{*}\right)+L^{2}\left(0, T ;\left(W^{1,2}\right)^{*}\right)$. Here, $q^{\prime}$ is the conjugate of $p$, and $\left(W^{1, q^{\prime}}\left(\mathbb{R}^{2}\right)\right)^{*}$ is the dual space for $W^{1, q^{\prime}}\left(\mathbb{R}^{2}\right)$. Indeed, for $\phi \in L^{q}\left(0, T ; W^{1, q}\right) \cap L^{q^{\prime}}(0$, $\left.T ;\left(W^{1, q^{\prime}}\left(\mathbb{R}^{2}\right)\right)\right) \cap L^{2}\left(0, T ;\left(W^{1,2}\left(\mathbb{R}^{2}\right)\right)\right)$ with $\nabla \cdot \phi=0$,

$$
\begin{aligned}
\int_{0}^{T} \int_{\mathbb{R}^{2}} \frac{d u^{m}}{d t} \cdot \phi d x d t= & \int_{0}^{T} \int_{\mathbb{R}^{2}}\left(\nabla \cdot S\left(D u^{m}\right) d x-\nabla \cdot\left(u^{m} \otimes u^{m}\right)+\nabla \cdot\left(b^{m} \otimes b^{m}\right)\right) \cdot \phi d x d t \\
= & -\int_{0}^{T} \int_{\mathbb{R}^{2}}\left|D u^{m}\right|^{q-2} D u^{m}: \nabla \phi d x d t+\int_{0}^{T} \int_{\mathbb{R}^{2}}\left(u^{m} \otimes u^{m}\right): \nabla \phi d x d t \\
& -\int_{0}^{T} \int_{\mathbb{R}^{2}}\left(b^{m} \otimes b^{m}\right): \nabla \phi d x d:=\mathscr{I}_{1}+\mathscr{I}_{2}+\mathscr{I}_{3} .
\end{aligned}
$$

(i) Estimate of $\mathscr{I}_{1}$ : using Hölder's inequality and the energy estimate (18), we have 


$$
\begin{aligned}
\left|I_{1}\right| & \leq\left\|\left|D u^{m}\right|^{q-1}\right\|_{L^{q^{\prime}}\left(0, T ; L^{q^{\prime}}\right)}\|\nabla \phi\|_{L^{q}\left(0, T ; L^{q}\right)} \leq\left\|D u^{m}\right\|_{L^{q}\left(0, T ; L^{q}\right)}^{q-1}\|\nabla \phi\|_{L^{q}\left(0, T ; L^{q}\right)} \\
& \leq C\left(\left\|u_{0}\right\|_{L^{2}\left(\mathbb{R}^{2}\right)}^{2}, T, q\right)\|\nabla \phi\|_{L^{q}\left(0, T ; L^{q}\right)^{q}} .
\end{aligned}
$$

(ii) Estimate of $\mathscr{I}_{2}$ : since $u^{m}$ belongs to $L^{2 q}\left(0, T ; L^{2 q}\right)$, we have

$$
\begin{aligned}
\left|I_{2}\right| & \leq\left\|u^{m} \otimes u^{m}\right\|_{L^{q}\left(0, T ; L^{q}\right)}\|\nabla \phi\|_{L^{q /(q-1)}\left(0, T ; L^{q /(q-1)}\right)} \\
& \leq\left\|u^{m}\right\|_{L^{2 q}\left(0, T ; L^{2 q}\right)}\left\|u^{m}\right\|_{L^{2 q}\left(0, T ; L^{2 q}\right)}\|\nabla \phi\|_{L^{q /(q-1)}\left(0, T ; L^{q /(q-1)}\right)} \leq C .
\end{aligned}
$$

(iii) Estimate of $\mathscr{I}_{3}$ : using Hölder's inequality, we have

$$
\begin{aligned}
\left|I_{3}\right| & \leq\left\|b^{m} \otimes b^{m}\right\|_{L^{2}\left(0, T ; L^{2}\right)}\|\nabla \phi\|_{L^{2}\left(0, T ; L^{2}\right)} \\
& \leq\left\|b^{m}\right\|_{L^{4}\left(0, T ; L^{4}\right)}\left\|b^{m}\right\|_{L^{4}\left(0, T ; L^{4}\right)}\|\nabla \phi\|_{L^{2}\left(0, T ; L^{2}\right)} \leq C .
\end{aligned}
$$

We combine (20), (21), and (22) to get

$\frac{d u^{m}}{d t} \in L^{q^{\prime}}\left(0, T ;\left(W^{1, q}\right)^{*}\right)+L^{q}\left(0, T ;\left(W^{1, q^{\prime}}\right)^{*}\right)+L^{2}\left(0, T ;\left(W^{1,2}\right)^{*}\right)$.

To obtain the distributive time derivative $d b^{m} / d t$, using the similar argument above, we have

$$
\frac{d b^{m}}{d t} \in L^{2}\left(0, T ;\left(W^{1,2}\right)^{*}\right)+L^{2}\left(0, T ;\left(W^{1,4}\right)^{*}\right)
$$
$=0$,

Indeed, for $\phi \in L^{2}\left(0, T ; W^{1,2}\right) \cap L^{2}\left(0, T ; W^{1,4}\right)$ with $\nabla \cdot \phi$

$$
\begin{aligned}
& \int_{0}^{T} \int_{\mathbb{R}^{2}} \frac{d u^{m}}{d t} \cdot \phi d x d t=-\int_{0}^{T} \int_{\mathbb{R}^{2}} \nabla b^{m} \\
& \quad: \nabla \phi d x d t+\int_{0}^{T} \int_{\mathbb{R}^{2}}\left(u^{m} \otimes b^{m}\right): \nabla \phi d x d t:=\mathscr{J}_{1}+\mathscr{J}_{2} .
\end{aligned}
$$

(iv) Estimate of $\mathscr{I}_{1}$ : using Hölder's inequality and the estimate (18), we have

$$
\left|\mathscr{J}_{1}\right| \leq\left\|\nabla b^{m}\right\|_{L^{2}\left(0, T ; L^{2}\right)}\|\nabla \phi\|_{L^{2}\left(0, T ; L^{2}\right)} \leq C .
$$

(v) Estimate of $\mathscr{I}_{2}$ : using Hölder's inequality, we have

$$
\left|\mathscr{J}_{2}\right| \leq C\left\|u^{m}\right\|_{L^{2 q}\left(0, T ; L^{2 q}\right)}\left\|b^{m}\right\|_{L^{4}\left(0, T ; L^{4}\right)}\|\nabla \phi\|_{L^{\frac{4 q}{q-2}}\left(0, T ; L^{\frac{4 q}{q-2}}\right)} \leq C .
$$

Due to the energy estimate (18) and time derivative class for $u^{m}$ and $b^{m}$, we can choose subsequences $u^{m_{k}}$ and $b^{m_{k}}$ such that

$$
\begin{gathered}
u^{m_{k}} \rightarrow u \text { weakly in } L^{\infty}\left(I ; L^{2}\left(\mathbb{R}^{2}\right)\right) \cap L^{q}\left(I ; W^{1, q}\left(\mathbb{R}^{2}\right)\right), \\
b^{m_{k}} \rightarrow b \text { weakly in } L^{\infty}\left(I ; L^{2}\left(\mathbb{R}^{2}\right)\right) \cap L^{2}\left(I ; W^{1,2}\left(\mathbb{R}^{2}\right)\right), \\
\partial_{t} u^{m_{k}} \rightarrow \partial_{t} u \text { weakly in } L^{q^{\prime}}\left(0, T ;\left(W^{1, q}\right)^{*}\right) \\
+L^{q}\left(0, T ;\left(W^{1, q^{\prime}}\right)^{*}\right)+L^{2}\left(0, T ;\left(W^{1,2}\right)^{*}\right),
\end{gathered}
$$

$\partial_{t} b^{m_{k}} \rightarrow \partial_{t} b$ weakly in $L^{2}\left(0, T ;\left(W^{1,2}\right)^{*}\right)+L^{2}\left(0, T ;\left(W^{1,4}\right)^{*}\right)$,

when $k$ goes to $\infty$. From the class of $u^{m_{k}}$ and $b^{m_{k}}$ in the convergence above and by the Aubin-Lions lemma (e.g., [20], Lemma 3.1), we have

$$
\begin{gathered}
u^{m_{k}} \rightarrow u \text { strongly in } L_{\mathrm{loc}}^{p}\left(\mathbb{R}^{2} \times I\right), p \in[1,2 q), \\
b^{m_{k}} \rightarrow b \text { strongly in } L_{\mathrm{loc}}^{\tilde{p}}\left(\mathbb{R}^{2} \times I\right), \tilde{p} \in[1,4) .
\end{gathered}
$$

Thus, we have

$$
\begin{aligned}
& u^{m_{k}} \rightarrow u \text { strongly in } L_{\text {loc }}^{2}\left(\mathbb{R}^{2} \times I\right), \\
& b^{m_{k}} \rightarrow b \text { strongly in } L_{\text {loc }}^{2}\left(\mathbb{R}^{2} \times I\right),
\end{aligned}
$$

as $k \rightarrow \infty$. So then, due to the weak and strong convergence above, it is possible to pass to the limit in the nonlinear terms (see, e.g., [21]). Moreover, $S\left(D u^{m}\right)$ is uniformly bounded in $L^{q^{\prime}}\left(\mathbb{R}^{2} \times(0, T)\right)$, and so $S(D u) \rightarrow A$ in this class. Hence, we will check $A=S(D u)$ which is shown by monotonicity trick (see [13], pp. 635-636). For this, we note that for $q \geq 2$,

$$
\begin{aligned}
t & \rightarrow \int_{\mathbb{R}^{2}}(u \cdot \nabla u) \cdot u d x \in L^{1}(0, T), t \rightarrow \int_{\mathbb{R}^{2}}(u \cdot \nabla b) \cdot b d x \in L^{1}(0, T) \\
t & \rightarrow \int_{\mathbb{R}^{2}}(b \cdot \nabla b) \cdot u d x \in L^{1}(0, T), t \rightarrow \int_{\mathbb{R}^{2}}(b \cdot \nabla u) \cdot b d x \in L^{1}(0, T) .
\end{aligned}
$$

From the energy equality, we have for $0 \leq s \leq T$

$$
\begin{aligned}
& \frac{1}{2}\left(\|u(s)\|_{L^{2}}^{2}+\|b(s)\|_{L^{2}}^{2}\right)+\int_{0}^{s}\|\nabla b\|_{L^{2}} d t+\int_{0}^{s} A \cdot D u d t \\
& \quad=\frac{1}{2}\left(\left\|u_{0}\right\|_{L^{2}}^{2}+\left\|b_{0}\right\|_{L^{2}}^{2}\right) .
\end{aligned}
$$


Define

$X_{s}^{m}=\int_{0}^{s}\left(S\left(D u^{m}\right)-S(D \phi), D u^{m}-D \phi\right) d t+\frac{1}{2}\|u(s)\|_{L^{2}}^{2}, \phi \in L^{q}\left(0, T ; W_{\sigma}^{1, q}\right)$.

Here, $W_{\sigma}^{1, q}:=\left\{v \in W^{1, q}\left(\mathbb{R}^{2}\right): \nabla \cdot v=0\right\}$. So, due to the property of the monotone operator $S$ and the semicontinuity of the norm, we obtain

$$
\liminf _{m \rightarrow \infty} X_{s}^{m} \geq \frac{1}{2}\|u(s)\|^{2}
$$

and also

$\lim _{m \rightarrow \infty} X_{s}^{m}=\int_{0}^{s} \frac{1}{2}\left\|u_{0}\right\|_{L^{2}}^{2}+\int_{0}^{s}(b \cdot \nabla b) \cdot u d t-\int_{0}^{s}(A, D \phi) d t-\int_{0}^{s}(S(D \phi), D u-D \phi) d t$

Then, due to the equality (32), we have

$$
\int_{0}^{s}(A-S(D \phi)) \cdot(D u-D \phi) d t \geq 0 \text {, a.e.s } \in[0, T] .
$$

Putting $\phi=u-\lambda w$ for $\lambda>0$ and $w \in L^{q}\left(0, T ; W_{\sigma}^{1, q}\right)$, we obtain

$$
\int_{0}^{s}(A-S(D u-\lambda w)) \cdot w d t \geq 0
$$

As $\lambda \rightarrow 0$, we deduce

$$
\int_{0}^{s}(A-S(D u)) \cdot w d t \geq 0
$$

which means that $A=S(D u)$ for a.e. $s \in[0, T]$. Hence, the proof of existence for weak solutions is completed.

Part B: uniqueness

For this part, we consider the equation for $v=u^{1}-u^{2}, h$ $=b^{1}-b^{2}$, and $\tilde{\pi}=\pi^{1}-\pi^{2}$ :

$$
\begin{aligned}
& \partial_{t} v-\nabla \cdot\left(\left(1+\left|D u^{1}\right|\right)^{q-2} D u^{1}\right)+\nabla \cdot\left(\left(1+\left|D u^{2}\right|\right)^{q-2} D u^{2}\right)+\left(u^{1} \cdot \nabla\right) v \\
& \quad+(v \cdot \nabla) u^{2}-\left(b^{1} \cdot \nabla\right) h-(h \cdot \nabla) b^{2}+\nabla \tilde{\pi}=0,
\end{aligned}
$$$$
\partial_{t} h-\Delta \tilde{h}+\left(u^{1} \cdot \nabla\right) h+(v \cdot \nabla) b^{2}-\left(b^{1} \cdot \nabla\right) v-(h \cdot \nabla) u^{2}=0,
$$

with $\operatorname{div} v=0$ and $\operatorname{div} h=0$. Testing $v$ and $h$ to the equations above, we have

$$
\begin{aligned}
& \frac{1}{2} \frac{d}{d t}\left(\|v\|_{L^{2}\left(\mathbb{R}^{2}\right)}^{2}+\|h\|_{L^{2}\left(\mathbb{R}^{2}\right)}^{2}\right)+\left(\|\nabla v\|_{L^{2}\left(\mathbb{R}^{2}\right)}^{2}+\|\nabla h\|_{L^{2}\left(\mathbb{R}^{2}\right)}^{2}\right) \\
& \quad \leq \int_{\mathbb{R}^{2}}(v \cdot \nabla) u^{2} \cdot v-\int_{\mathbb{R}^{2}}(h \cdot \nabla) b^{2} \cdot v+\int_{\mathbb{R}^{2}}(v \cdot \nabla) b^{2} \cdot h-\int_{\mathbb{R}^{2}}(h \cdot \nabla) u^{2} \cdot h \\
& \leq\|v\|_{L^{2}}^{2}\left\|u^{2}\right\|_{L^{2}}^{2}\left\|\nabla u^{2}\right\|_{L^{2}}^{2}+\|h\|_{L^{2}}^{2}\left\|b^{2}\right\|_{L^{2}}^{2}\left\|\nabla b^{2}\right\|_{L^{2}}^{2}+\|v\|_{L^{2}}^{2}\left\|b^{2}\right\|_{L^{2}}^{2}\left\|\nabla b^{2}\right\|_{L^{2}}^{2} \\
& \quad+\|h\|_{L^{2}}^{2}\left\|u^{2}\right\|_{L^{2}}^{2}\left\|\nabla u^{2}\right\|_{L^{2}}^{2}+\frac{1}{2}\left(\|\nabla v\|_{L^{2}}^{2}+\|\nabla h\|_{L^{2}}^{2}\right),
\end{aligned}
$$

that is,

$$
\begin{aligned}
& \frac{1}{2} \frac{d}{d t}\left(\|v\|_{L^{2}\left(\mathbb{R}^{2}\right)}^{2}+\|h\|_{L^{2}\left(\mathbb{R}^{2}\right)}^{2}\right)+\left(\|\nabla v\|_{L^{2}\left(\mathbb{R}^{2}\right)}^{2}+\|\nabla h\|_{L^{2}\left(\mathbb{R}^{2}\right)}^{2}\right) \\
& \quad \leq\left(\|v\|_{L^{2}}^{2}+\|h\|_{L^{2}}^{2}\right)\left(\left\|u^{2}\right\|_{L^{2}}^{2}\left\|\nabla u^{2}\right\|_{L^{2}}^{2}+\left\|b^{2}\right\|_{L^{2}}^{2}\left\|\nabla b^{2}\right\|_{L^{2}}^{2}\right) .
\end{aligned}
$$

Applying Gronwall's inequality, we obtain $v=0$ and $h=0$ in $\mathbb{R}^{2}$ and hence $u_{1}=u_{2}$ and $b_{1}=b_{2}$.

Part C: decay rate

A proof of this part is almost the same as that in [17]. For the convenience of the reader, it gives a proof. From the $L^{2}$ -energy inequality and Korn's inequality, it follows that

$$
\frac{1}{2} \frac{d}{d t} \int_{\mathbb{R}^{3}}\left(|u|^{2}+|b|^{2}\right) d x+\min \{C, 1\} \int_{\mathbb{R}^{3}}\left(|\nabla u|^{2}+|\nabla b|^{2}\right) \leq 0,
$$

where $C>0$ is a Korn-type constant. Applying Plancherel's theorem to (42) yields

$$
\begin{aligned}
& \frac{1}{2} \frac{d}{d t} \int_{\mathbb{R}^{3}}\left(|u \wedge(\xi, t)|^{2}+|b \wedge(\xi, t)|^{2}\right) d \xi+\min \{C, 1\} \int_{\mathbb{R}^{3}}|\xi|^{2} \\
& \cdot\left(|u \wedge(\xi, t)|^{2}+|b \wedge(\xi, t)|^{2}\right) d \xi \leq 0 .
\end{aligned}
$$

Put $\Phi(\xi, t):=|u \wedge(\xi, t)|^{2}+|b \wedge(\xi, t)|^{2}$. Let $f(t)$ be a smooth function of $t$ with $f(0)=1, f(t)>0$ and $f^{\prime}(t)>0$. Set $S(t)=\left\{\xi \in \mathbb{R}^{n}: 2 \min \{C, 1\} f(t)|\xi|^{2} \leq f^{\prime}(t)\right\}$. Then,

$$
\begin{aligned}
& 2 \min \{C, 1\} f(t) \int_{\mathbb{R}^{3}}|\xi|^{2}|\Phi(\xi, t)|^{2} d \xi \\
& \quad \geq f^{\prime}(t) \int_{\mathbb{R}^{3}}|\Phi(\xi, t)|^{2} d \xi-f^{\prime}(t) \int_{S(t)}|\Phi(\xi, t)|^{2} d \xi .
\end{aligned}
$$

Since

$$
\begin{aligned}
& \frac{d}{d t}\left(f(t) \int_{\mathbb{R}^{3}}|\Phi(\xi, t)|^{2} d \xi\right)+2 \min \{C, 1\} f(t) \int_{\mathbb{R}^{3}}|\xi|^{2}|\Phi(\xi, t)|^{2} d \xi \\
& \quad \leq f^{\prime}(t) \int_{\mathbb{R}^{3}}|\Phi(\xi, t)|^{2} d \xi,
\end{aligned}
$$


we have

$\frac{d}{d t}\left(f(t) \int_{\mathbb{R}^{n}}|u \wedge(\xi, t)|^{2} d \xi\right) \leq f^{\prime}(t) \int_{S(t)}|u \wedge(\xi, t)|^{2} d \xi$

Integrating in time, we get

$$
\begin{aligned}
& f(t) \int_{\mathbb{R}^{n}}\left(|u \wedge(\xi, t)|^{2}+|b \wedge(\xi, t)|^{2}\right) d \xi \\
& \leq \int_{\mathbb{R}^{n}}\left(\left|u \wedge_{0}(\xi)\right|^{2}+\left|b \wedge_{0}(\xi)\right|^{2}\right) d \xi+C \int_{0}^{t} f^{\prime}(s) \int_{S(s)} \\
& \quad \times\left(|u \wedge(\xi, s)|^{2}+|b \wedge(\xi, s)|^{2}\right) d \xi d s .
\end{aligned}
$$

Set $f(t)=(1+t)^{2}$. From Lemma 3 with Young's inequality and the energy estimate, we have

$$
\begin{aligned}
& (1+t)^{2} \int_{\mathbb{R}^{n}}\left(|u \wedge(\xi, t)|^{2}+|b \wedge(\xi, t)|^{2}\right) d \xi \\
& \leq \int_{\mathbb{R}^{n}}\left(\left|u \wedge_{0}(\xi)\right|^{2}+\left|b \wedge_{0}(\xi)\right|^{2}\right) d \xi+C \int_{0}^{t}(1+s) \int_{S(s)} \\
& \times\left(\left|u \wedge_{0}(\xi)\right|^{2}+\left|b \wedge_{0}(\xi)\right|^{2}\right) d \xi d s+C \int_{0}^{t}(1+s) \int_{S(s)}|\xi|^{2} \\
& \times\left(\int_{0}^{t}\left(\|u(s)\|_{L^{2}}^{2}+\|b(s)\|_{L^{2}}^{2}\right) d s\right)^{2} d \xi d s \\
& +C \int_{0}^{t}(1+s) \int_{S(s)}|\xi|^{2}\left(\int_{0}^{t}\|u(s)\|_{L^{2}\left(\mathbb{R}^{2}\right)}^{2} d s\right)^{1 /(p-1)} d \xi d s \\
& \leq \int_{\mathbb{R}^{n}}\left(\left|u \wedge_{0}(\xi)\right|^{2}+\left|b \wedge_{0}(\xi)\right|^{2}\right) d \xi+C \int_{0}^{t}(1+s) \int_{S(s)} \\
& \times\left(\left|u \wedge_{0}(\xi)\right|^{2}+\left|b \wedge_{0}(\xi)\right|^{2}\right) d \xi d s+C \int_{0}^{t}(1+s) \int_{S(s)} s|\xi|^{2} \\
& \times\left(\int_{0}^{t}\left(\|u(s)\|_{L^{2}}^{4}+\|b(s)\|_{L^{2}}^{4}\right) d s\right) d \xi d s \\
& +C \int_{0}^{t}(1+s) \int_{S(s)} s|\xi|^{2}\left(\int_{0}^{t}\|u(s)\|_{L^{2}\left(\mathbb{R}^{2}\right)}^{2} d s+C\right) d \xi d \\
& \leq \int_{\mathbb{R}^{n}}\left(\left|u \wedge_{0}(\xi)\right|^{2}+\left|b \wedge_{0}(\xi)\right|^{2}\right) d \xi+C \int_{0}^{t}(1+s) \int_{S(s)} \\
& \times\left(\left|u \wedge_{0}(\xi)\right|^{2}+\left|b \wedge_{0}(\xi)\right|^{2}\right) d \xi d \\
& +C \int_{0}^{t}(1+s)^{2} \int_{S(s)}|\xi|^{2} \int_{0}^{t}\left(\|u(s)\|_{L^{2}}^{4}+\|b(s)\|_{L^{2}}^{4}\right) d s d \xi d s \\
& +C \int_{0}^{t}(1+s)^{2} \int_{S(s)}|\xi|^{2} \int_{0}^{t}\|u(s)\|_{L^{2}\left(\mathbb{R}^{2}\right)}^{2} d s d \xi d s \\
& \leq C+C(1+t)+\left(\int_{0}^{t}\left(\|u(s)\|_{L^{2}}^{2}+\|b(s)\|_{L^{2}}^{2}\right) d s\right) \text {. }
\end{aligned}
$$

Thus, we get

$$
\begin{aligned}
& (1+t) \int_{\mathbb{R}^{3}}\left(|u(\xi, t)|^{2}+|b(\xi, t)|^{2}\right) d \xi \\
& \leq C+C \int_{0}^{t}(1+s)\left(\|u(s)\|^{2}+\|b(s)\|^{2}\right)(1+s)^{-1} d s
\end{aligned}
$$
that

Applying Gronwall's inequality, we immediately deduce

$$
\|u(t)\|_{L^{2}}+\|b(t)\|_{L^{2}} \leq C(1+t)^{-1 / 2}
$$

thus, we finally obtain the desired result.

\section{Appendix}

Here, we mention the existence of unique strong solution for (1)-(2). Its proof is easily checked from the argument in [15] or [22]. And thus, we omit the proof.

Definition A.1. Let $2<q<\infty$ and $\mu_{0} \geq 0$ and $\mu_{1}>0$. Suppose that $u_{0} \in\left(W^{1,2} \cap W^{1, q}\right)\left(\mathbb{R}^{2}\right)$ and $b_{0} \in W^{1,2}\left(\mathbb{R}^{2}\right)$. We say that a weak solution $(u, b)$ is a strong solution to the incompressible 2D-MHD equations of non-Newtonian fluids (1)-(2) if

$$
\begin{gathered}
\nabla u \in L^{\infty}\left(0, T ; L^{q} \cap L^{2}\left(\mathbb{R}^{2}\right)\right), \\
b \in L^{\infty}\left(0, T ; W^{1,2}\left(\mathbb{R}^{2}\right)\right) \cap L^{2}\left(0, T ; W^{2,2}\left(\mathbb{R}^{2}\right)\right), \\
u_{t}, b_{t} \in L^{2}\left(Q_{T}\right), S(D u) \in L^{q^{\prime}}\left(0, T ; W_{\operatorname{loc}}^{1, q^{\prime}}\left(\mathbb{R}^{2}\right)\right) . \\
\int_{0}^{T} \int_{\mathbb{R}^{2}}|D u|^{q-2}\left|D^{2} u\right|^{2} d x d t<\infty .
\end{gathered}
$$

Here, $q^{\prime}$ means the Hölder conjugate of $q$.

Theorem A.2. Let $2<q<\infty$ and $\mu_{0} \geq 0$ and $\mu_{1}>0$. Suppose that $u_{0} \in\left(W^{1,2} \cap W^{1, q}\right)\left(\mathbb{R}^{2}\right)$ and $b_{0} \in W^{1,2}\left(\mathbb{R}^{2}\right)$. Then, there exists a strong solution $(u, b)$ of the incompressible $2 D-M H D$ equations of the non-Newtonian type (1)-(2) in the sense of Definition A.1.

\section{Data Availability}

This paper uses the method of theoretical analysis.

\section{Conflicts of Interest}

The author declares that he has no conflicts of interest.

\section{Acknowledgments}

Jae-Myoung Kim was supported by the National Research Foundation of Korea Grant funded by the Korean Government (NRF-2020R1C1C1A01006521).

\section{References}

[1] S. Whitaker, Introduction to Fluid Mechanics, Krieger, 1986. 
[2] W. L. N. Wilkinson, On-Newtonian Fluids: Fluid Mechanics, Mixing and Heat Transfer, Pergamon Press, London. New York, 1960.

[3] H. I. Andersson, K. H. Bach, and B. S. Dandapat, "Magnetohydrodynamic flow of a power-law fluid over a stretching sheet," International Journal of Non-Linear Mechanics, vol. 27, no. 6, pp. 929-936, 1992.

[4] T. Sarpakaya, "Flow of non-Newtonian fluids in a magnetic field," AICHE Journal, vol. 7, no. 2, pp. 324-328, 1961.

[5] Y. G. Sapunkov, "Self-similar solutions of non-Newtonian fluid boundary layer in MHD," Fluid Dynamics, vol. 2, pp. 42-47, 1967.

[6] G. Bohme, "Non-Newtonian fluid mechanics," North-Holland Series in Applied Mathematics and Mechanics, vol. 31, 1987.

[7] O. A. Ladyzhenskaya, "New equations for the description of the motions of viscous incompressible fluids, and global solvability for their boundary value problems," Trudy Matematicheskogo Instituta Imeni VA Steklova, vol. 102, pp. 85-104, 1967.

[8] O. A. Ladyzhenskaya, The Mathematical Theory of Viscous Incompressible Flow, Gordon and Breach, New York, NY, USA, 2nd edition, 1969.

[9] J.-L. Lions, Quelques Méthodes de Résolution des Problèmes aux Limites non Linéaires, Dunod, Paris, 1969.

[10] L. Diening, M. Råžička, and J. Wolf, "Existence of weak solutions for unsteady motions of generalized Newtonian fluids," Annali della Scuola Normale Superiore di Pisa - Classe di Scienze, vol. 5, pp. 1-46, 2010.

[11] J. Wölf, "Existence of weak solutions to the equations of nonstationary motion of non-Newtonian fluids with shear rate dependent viscosity," Journal of Mathematical Fluid Mechanics, vol. 9, pp. 104-138, 2007.

[12] L. C. F. Ferreira and E. J. Villamizar-Roa, "Exponentially-stable steady flow and asymptotic behavior for the magnetohydrodynamic equations," Communications in Mathematical Sciences, vol. 9, no. 2, pp. 499-516, 2011.

[13] V. N. Samokhin, "On a system of equations in the magnetohydrodynamics of nonlinearly viscous media," Differential Equations, vol. 27, no. 5, pp. 628-636, 1991.

[14] V. N. Samokhin, "Existence of a solution of a modification of a system of equations of magnetohydrodynamics," Mathematics of the USSR-Sbornik, vol. 72, no. 2, pp. 373-385, 1992.

[15] M. D. Gunzburger, O. A. Ladyzhenskaya, and J. S. Peterson, "On the global unique solvability of initial-boundary value problems for the coupled modified Navier-Stokes and Maxwell equations," Journal of Mathematical Fluid Mechanics, vol. 6, no. 4, pp. 462-482, 2004.

[16] P. A. Razafimandimby, "Trajectory attractor for a nonautonomous magnetohydrodynamic equation of nonNewtonian fluids," Dynamics of Partial Differential Equations, vol. 9, no. 3, pp. 177-203, 2012.

[17] J.-M. Kim, "Temporal decay of strong solutions to the magnetohydrodynamics with power-law type nonlinear viscous fluid," Journal of Mathematical Physics, vol. 61, no. 1, article 011504, p. 6, 2020.

[18] P. Milan, "Cauchy problem for the non-Newtonian viscous incompressible fluid," Applications of Mathematics, vol. 41, pp. 169-201, 1996.

[19] J. Málek, J. Nečas, M. Rokyta, and M. Růžička, Weak and Measure-Valued Solutions to Evolutionary PDEs, Chapman \& Hall, 1996
[20] H. Bellout, F. Bloom, and J. Nečas, "Solutions for incompressible non-Newtonian fluids," Comptes rendus de l'Académie des sciences, Série 1: Mathématique, vol. 317, pp. 795-800, 1993.

[21] H. Kozono, "Weak and classical solutions of the two dimensional magnetohydrodynamic equations," Tohoku Mathematical Journal, Second Series, vol. 51, no. 2, pp. 471-488, 1989.

[22] K. Kang and J.-M. Kim, "Existence of solutions for the magnetohydrodynamics with power-law type nonlinear viscous fluid," Nonlinear Differential Equations and Applications, vol. 26, no. 11, p. 24, 2019. 